\title{
Synchrotron X-Ray Microdiffraction Studies of Electromigration in Interconnect lines at the Advanced Light Source
}

\author{
N. Tamura, K. Chen and M. Kunz \\ Advanced Light Source, Lawrence Berkeley Laboratory, 1 Cyclotron Road, Berkeley CA \\ 94720, USA.
}

\begin{abstract}
Synchrotron polychromatic X-ray microdiffraction is a particularly suitable technique to study in situ the effect of electromigration in metal interconnects as add spatial resolution to grain orientation and strain sensitivity. This technique has been extensively used at the Advanced Light Source to monitor changes in aluminum and copper interconnect test structures while high-density current is passed into them during accelerated tests at elevated temperature. One of the principal findings is the observation of electromigrationinduced plasticity in the metal lines that appear during the very early stages of electromigration. In some of the lines, high density of geometrically necessary dislocation are formed leading to additional diffusion paths causing an enhancement of electromigration effect at test temperature.
\end{abstract}

\section{Introduction}

In situ spatially resolved studies of electromigration (EM) effects on metal interconnect lines have been one of the major projects that have been conducted on the X-ray microdiffraction end station formerly located on the 7.3.3 bending magnet beamline at the Advanced Light Source (ALS). The initial funding of the end-station equipments have indeed been provided by Intel Corporation after the initial successful proof-ofprinciple microLaue experiments performed using the ALS then newly developed Kirkpatrick-Baez (KB) focusing mirror system [1]. The submicron size white beam spot size obtained by the KB optics allows for precisely measuring crystal orientation of individual grains in encapsulated aluminum interconnects [2]. This was followed by successful collaborations with Lucent Technologies and Advanced Micro Devices. The ability to obtain quantitative information such as crystal orientation and strain/stress at the individual grain level during in-situ tests made white beam X-ray microdiffraction a particularly useful tool to study mechanical properties of materials at this length scale, and make particularly suited to obtain a thorough understanding of EM effects on micron to submicron size buried interconnect structures. Besides the ALS, this type of study has been also carried out independently at the Advanced Photon Source (APS) [3] [4] and at the National Synchrotron Light Source (NSLS) [5].

This paper presents an overview of the principal results obtained from X-ray microdiffraction studies of electromigration effects on aluminum and copper 
interconnects at the ALS throughout continuous efforts that spanned over a decade (19982008) from approximately 40 weeks of combined beamtime.

\section{Synchrotron-based X-Ray Microdiffraction}

A polychromatic x-ray diffraction pattern (Laue pattern) taken with an area detector provides a wealth of structural information about the x-ray illuminated area of the sample. The indexing of the reflections in the pattern provides the shape of the unit cell and its orientation. Moreover small shifts of the reflection positions from their ideal "unstrained" positions provide the elastic deformation state of the unit cell at constant volume (deviatoric strain tensor). The total strain tensor can additionally be obtained by calculating its dilatational component via the measurement of the wavelength of one reflection. [6] The shape of the reflections provides some information about plasticity and the type and distribution of dislocations can be inferred. [7]. For x-ray fluorescing material, Kossel lines can be analyzed to derive strain as well [8].

The potential of Laue diffraction for microstructural studies becomes particularly useful when the x-ray beam size is smaller than the constitutive grain size of a polycrystalline material. Submicron size polychromatic beam are now routinely provided on dedicated beamlines at synchrotron facilities using achromatic focusing optics such as KirkpatrickBaez mirrors. [9] [10] Used in scanning mode (the sample is raster-scanned under the $\mathrm{x}$ ray microbeam and a Laue pattern is taken at each scan step), polychromatic x-ray microdiffraction can effectively be used to map grain orientation, strain and/or dislocation distribution in polycrystalline materials with submicron spatial resolution. [11] (Figure 1).

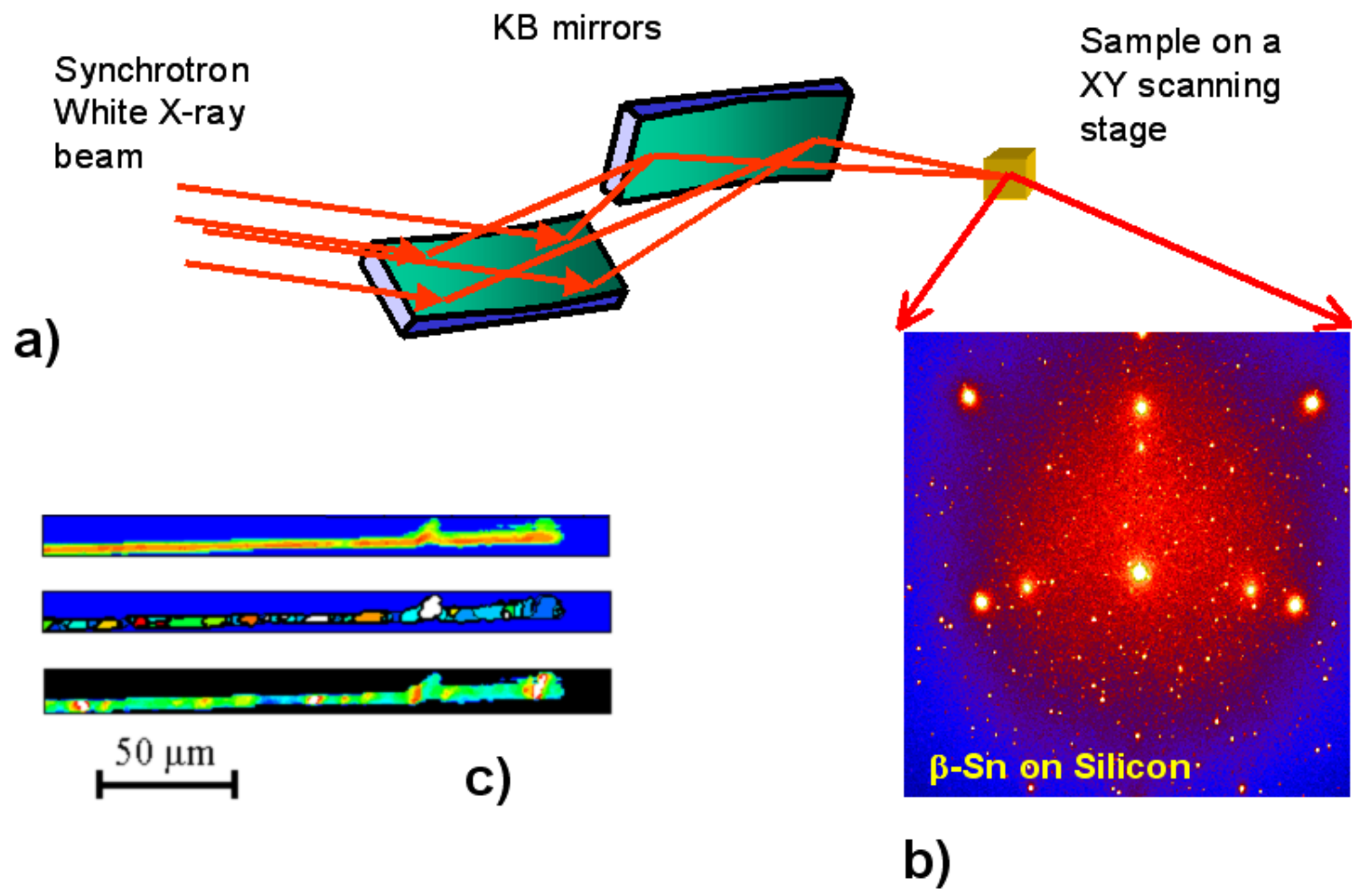


Figure 1.- Polychromatic (white) beam X-ray microdiffraction used in scanning mode is a powerful tool to study the microstructure of polycrystalline sample. Polychromatic X-ray beam from a synchrotron source are ultimately focused to submicron size using a pair of elliptically bent Kirkpatrick-Baez mirrors (a). The sample is raster-scanned under the beam and a Laue diffraction pattern is taken at each step (b). The analysis of the array of diffraction patterns provides grain orientation (c middle) and strain (c bottom) maps of the sample. $X$-ray fluorescence signal can also be collected providing an elemental distribution map of the sample (c top). The example show maps of a $200 \mu \mathrm{m}$ long $4 \mu \mathrm{m}$ wide $2 \mu \mathrm{m}$ thick wire of a $\beta$-Sn stripe deposited on silicon obtained by scanning the sample with a $500 \mathrm{~nm}$ step size.

The X-ray microdiffraction facility at the ALS, which was formerly operating on bending beamline 7.3.3, has recently been moved onto the superconducting magnet beamline 12.3.2. These beamlines have been described in details elsewhere [12] [13] and only a brief summary is given here. The white beam x-ray spectrum is primarily focused to the entrance of the hutch via a toroidal mirror. A pair of slits placed at the focal point act as an adjustable source size, which is further focused by a pair of elliptically bent Kirkpatrick-Baez mirrors to submicron sizes. A four-bounce monochromator consisting of two channel cuts rotating in opposite direction allows switching between white and monochromatic beam while illuminating the same spot on the sample. The sample is placed on a high precision XY stage and diffraction patterns are collected using an area detector (MAR133). Typical orientation resolution in the order of $0.01^{\circ}$ and strain resolution in the order of $10^{-4}$ can be achieved by this technique. The sample stage can be equipped with a heating device allowing to perform accelerated electromigration tests.

\section{Electromigration in passivated $\mathrm{Al}(\mathrm{Cu})$ interconnect lines}

In aluminum lines, electromigration occurs though grain boundary diffusion. In the case of polycrystalline lines where many grains span across the lines, the path for diffusion is continuous. This will result in the development of an electromigration-induced stress gradient along the line, with a tensile stress at the cathode end where material is depleted and a compressive stress at the anode end where the material is accumulated. [14] [15] [16] For lines below a certain critical length (Blech length), the stress gradient will eventually counterbalance the effect of electromigration as it creates a chemical potential causing the material to diffuse in the opposite direction, and no electromigration damage is observed (concept of immortal lines). Lines of length above the Blech length are subject to failure either by open circuit at the cathode or short circuit at the anode, when the compressive stress cause a crack in the passivation layer and the metal is extruded and comes into contact with neighboring metallizations. [5] [17] The situation is more complicated in the case of bamboo and near-bamboo structure where some or all the grains span the width of the line. In that case no continuous diffusion path is available for electromigration and these lines are generally more resistant to electromigration than polycrystalline lines. However failure above the critical length still occurs, indicating that a different EM diffusion process is at play. In order to elucidate electromigration failure mechanism in bamboo and near bamboo structures, B. C. Valek and co-workers performed microdiffraction studies with the initial goal to measure stress gradient as it was performed for large polycrystalline lines. The samples were encapsulated $\mathrm{Al}(\mathrm{Cu})$ interconnect lines of length $30 \mathrm{um}$, width $4 \mathrm{um}$ and thickness $0.7 \mathrm{um}$. The accelerated test 
experiment has been conducted over a period of several hours at a temperature of $224{ }^{\circ} \mathrm{C}$ and a current density approaching $1 \mathrm{MA} / \mathrm{cm}^{2}$. Results showed a very inhomogeneous stress distribution exhibiting local stress gradients, which are indicative of flux divergence points along the line, superimposing to the global stress gradient. This inhomogeneous stress distribution locally leads to the yielding of the material when the yield stress is reached. This is evidenced by streaking of the aluminum grain reflections in the Laue patterns, which are indicative of the presence of geometrically necessary dislocations (GNDs) which are nucleated to accommodate the diffused atoms or vacancies into the lattice. (Figure 2)

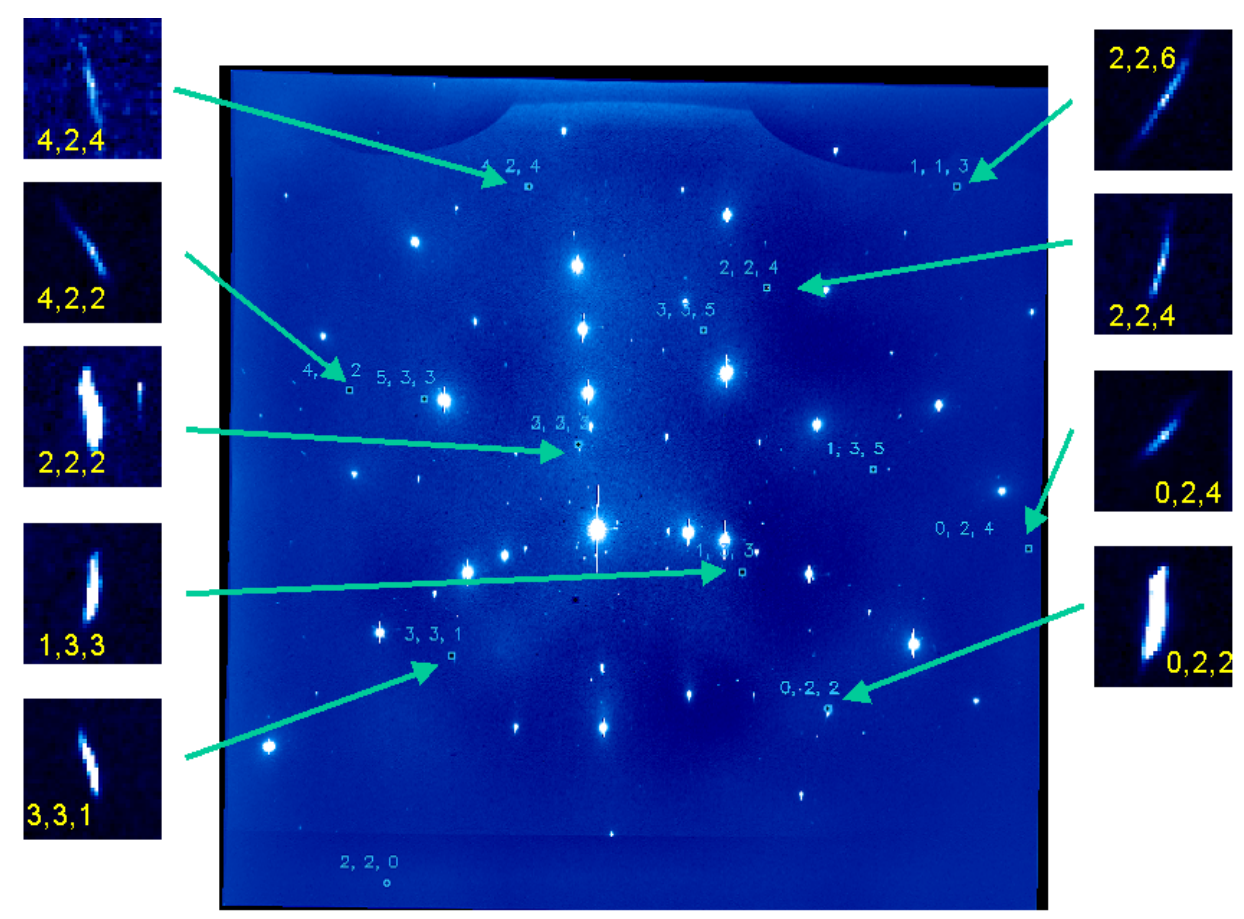

Figure 2.- Aluminum reflections are streaked in this Laue pattern of an aluminum grain in $\mathrm{Al}(\mathrm{Cu})$ interconnect sample subjected to electromigration by a current density of $1 \mathrm{MA} / \mathrm{cm} 2$ for 25 hours. Most of the bright spots belong to the silicon wafer. The comparatively weaker aluminum peaks are zoomed in on the sides of the Laue pattern.

Streakings appear at the very early stage of electromigration and their lengths can reach a few degrees. The amount of streaking depends on the position of the grain within the length of the line: it is the largest towards the cathode end of the line. The streaking direction is mainly perpendicular to the current flow direction. As the current pass, the streaked peaks of the largest grains decomposed into well-defined maxima, indicative of subgrain formation (polygonization). The amount of streaking also depends on the size of the grains. Small grains do not exhibit streaking but simply rotate around an axis in the direction of the current flow. [18] [19]

The direction of the peak streaking reflects the active dislocation glide system within the material. It is found that the first dislocations to participate to the electromigrationinduced grain deformation are the edge dislocations, which have their line direction the closest to the current flow. For higher tensile stress (grain position closer to the cathode 
end), a second dislocation glide system can become activated. At the cathode end, multiple glide systems can occur leading to complex shape Laue peaks (figure 3) [20] The X-ray microdiffraction data gives therefore a model of diffused atoms or vacancies accommodation for almost bamboo structure where bamboo grains deform and polygonize, while non-bamboo ones, simply rotate. Both the deformation and the rotations are compatible with a concave bending of the line across the direction of the current flow. The peak streaking associated with the deformation of the bamboo grains can be explained in term of geometrically necessary dislocations with line direction crystallographically the closest to the current flow direction. These dislocations ultimately form a network of possible diffusion path that could relays the grain boundary diffusion of polycrystalline interconnects.

\section{Cathode Anode}
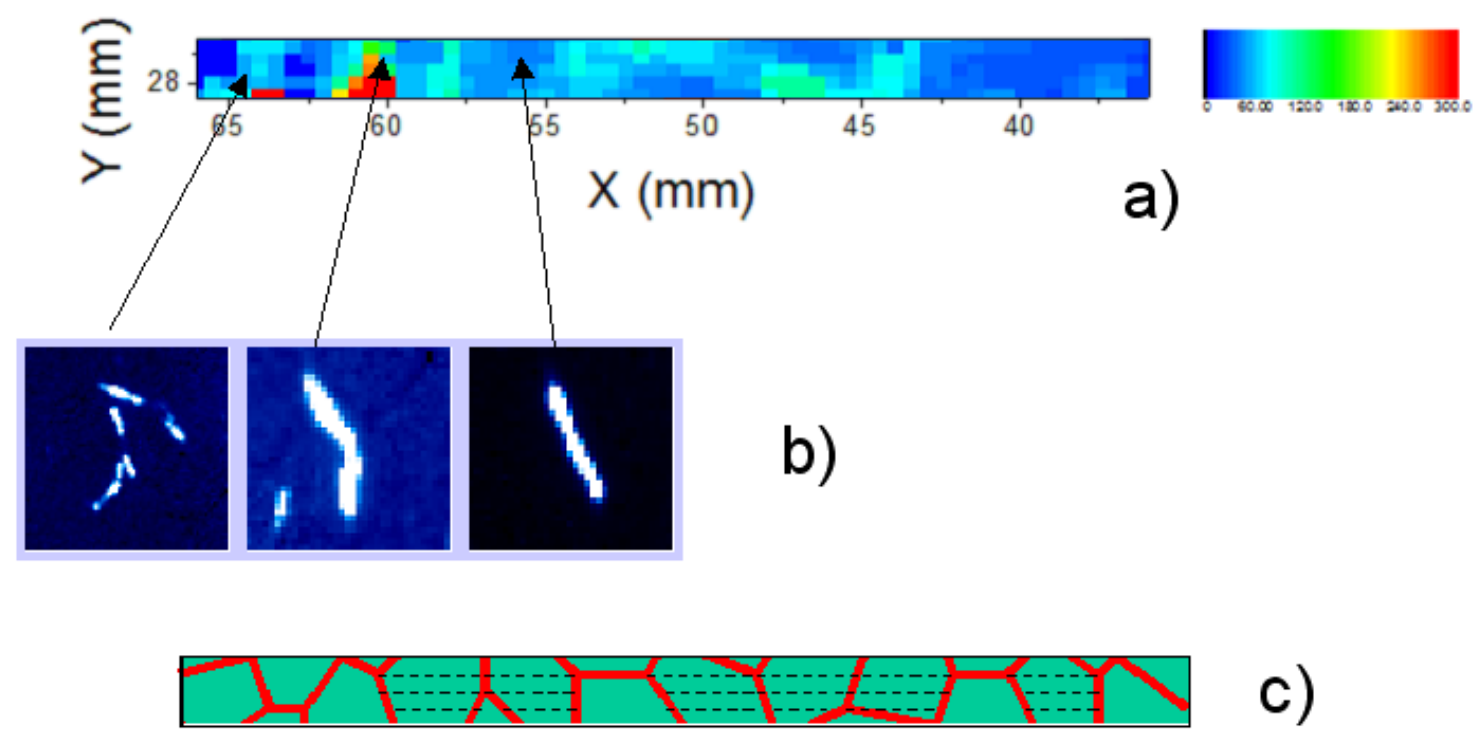

Figure 3.- a) This experimental maximum resolved shear stress (MRSS) map of a 30 um long, 4 um wide $\mathrm{Al}(\mathrm{Cu})$ interconnect after 25 hours of electromigration at $1 \mathrm{MA} / \mathrm{cm} 2$, shows an inhomogeneous stress distribution which superimposes to a global stress gradient. The MRSS and thus deformation is the highest at the cathode end. $b$ ) the shape of a Laue reflection gives an indication of the dislocation glide system participating to the deformation. For moderate stress, a single active glide system is present. At higher stress, a second glide system can be activated, while at the very end of the cathode, multiple glide systems occur simultaneously. c) $x$-ray microdiffraction analysis shows that this electromigration generated dislocation network, with line direction mostly parallel to the current flow could constitute besides grain boundaries, additional atomic diffusion path (the figure shows a schematic).

\section{Electromigration in Damascene Copper Interconnects}

Surface and interface diffusion are the main electromigration diffusion path for narrow copper interconnect structures. [21] Experiments have been performed on 300 um long, $1.1 \mathrm{um}$ wide and $0.7 \mathrm{um}$ thick lines, at a temperature of $355^{\circ} \mathrm{C}$ with a current density of $6.5 \mathrm{MA} / \mathrm{cm}^{2}$. While the aluminum interconnects line had an essentially (111) fiber texture, the damascene copper lines exhibit a more complex microstructure with twinning 
and sidewall components [22]. The results of the in-situ electromigration experiment show an essentially bamboo structure with inhomogeneous stress indicating a succession of accumulation and depletion areas in the vicinity of (115) twins (figure 4). This indicates that the boundaries between some (115) twin grains and (111) grains act as flux divergence points and this can be explained by the anisotropy of copper surface diffusivity. While (111) surface are essentially isotropic, (115) surface appear anisotropic with very different diffusivity values along $\left(4.410^{-7} \mathrm{~cm}^{2} / \mathrm{s}\right)$ and perpendicularly $\left(1.710^{-7}\right.$ $\mathrm{cm}^{2} / \mathrm{s}$ ) to the $<1,-1,0>$ directions. [23]

a)

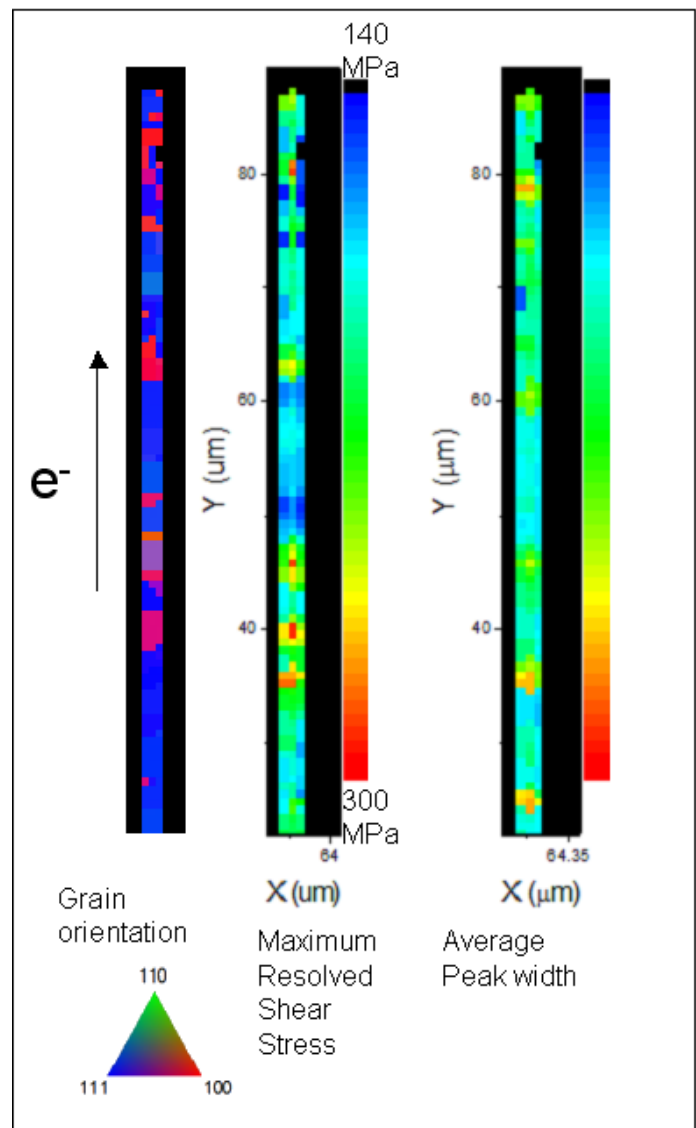

b)

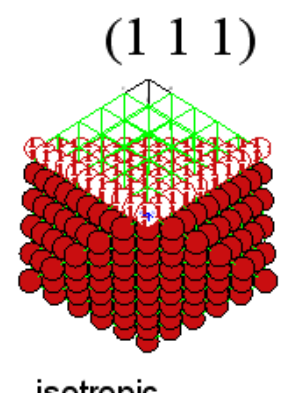

isotropic

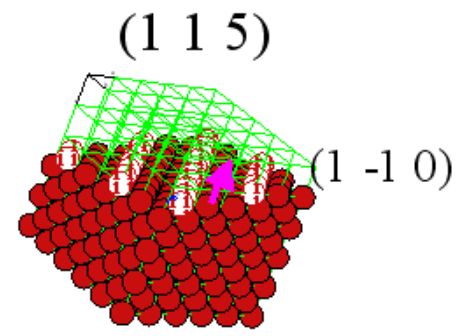

$\mathrm{D}_{i j}=4.410^{-7} \mathrm{~cm}^{2} / \mathrm{s}$

$D_{\perp}=1.710^{-7} \mathrm{~cm}^{2} / \mathrm{s}$

Figure 4.- a) Grain orientation, maximum resolved shear stress and average peak width maps obtained from the anode end of a 300 um long, 1.1 um wide and 0.7 um thick damascene copper line electromigrated under a current density of $6.5 \mathrm{MA} / \mathrm{cm} 2$. b) The resulting inhomogeneous stress distribution can be explained by anisotropy of the grain top surface diffusivity where (115) twin grain can act as blocking grain when their $<1,-1,0>$ atomic rows are perpendicular to the current flow.

The local increase in the average $\mathrm{x}$-ray reflection width indicates that plastic deformation also occurs in electromigrated copper interconnects, as in aluminum lines. This plastic deformation depends on the microtexture of the sample and can reach extreme proportions. This is the case for the 200 um long, 0.48 um wide and 0.175 um thick samples studied by A. S. Budiman and co-workers. Experiment was performed at $300{ }^{\circ} \mathrm{C}$ with a current density of $2 \mathrm{MA} / \mathrm{cm}^{2}$. The results are similar to the case of $\mathrm{Al}(\mathrm{Cu})$ with peak streaking appearing to be mainly perpendicular to the current direction and 
compatible with GNDs with their line direction along the $<112>$ crystallographic direction the closest to the current flow. The difference is that peak streaking appears both at the anode and the cathode ends with no obvious gradient of streak lengths as in the aluminum line case. This observation in itself is interesting as it raises the possibility of alleviating electromigration effects by controlling the texture of the material. [24] [25] Moreover, as in $\mathrm{Al}(\mathrm{Cu})$ interconnects, streaked Laue reflections are observed in many grains along the lines indicating the formation of a network of almost parallel dislocation lines along the current flow direction. The measured dislocation density $\left(\sim 2.10^{15} \mathrm{~m}^{-2}\right)$ at test temperature is high enough that the effect of the grain boundaries perpendicular to the current flow can be neglected, and that dislocation core diffusion outweighs surface/interface diffusion (figure 5).

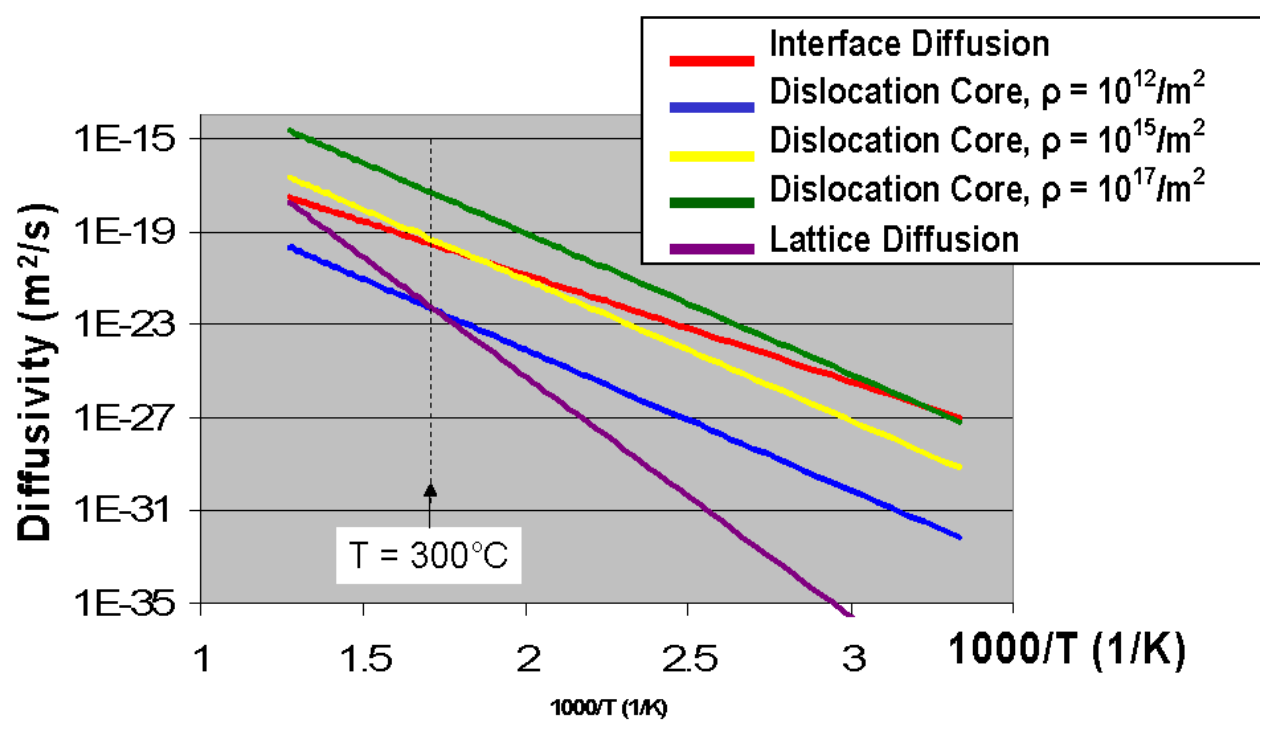

Figure 5.- Plot of diffusivity vs temperature for different diffusion mechanisms in copper. Dislocation core diffusion is dominant over interface diffusion at temperature above $300{ }^{\circ} \mathrm{C}$ and dislocation density in the order of $10^{15} \mathrm{~m}^{-2}$. At service temperature however, interface diffusion is dominant.

As dislocation core diffusion is not the dominant diffusion process at service temperature, these findings raises the possibility of serious consequences on the evaluation of interconnect lifetime, with an overestimation of the Mean Time to Failure (MTF) values measured from accelerated tests performed at high current density. [25] Black's equation is expressed as:

$$
M T F=A\left(\frac{1}{j}\right)^{n} \exp \left(\frac{E_{A}}{k T}\right) \text { (eq 1) }
$$

with $n$ equal to 1 at low current density and $\mathrm{n}>1$ at high current density. For a given test temperature, plasticity only occurs at high current density above a certain threshold which depends on the initial microstructural state of the interconnect line. This threshold is higher for samples, which are already plastically deformed, while the MTF curve shifts down. This further demonstrates that plasticity enhanced diffusivity plays a nonnegligible role in the estimation of device lifetime. [25] [26] 


\section{Conclusions}

Electromigration-induced plastic deformation in $\mathrm{Al}(\mathrm{Cu})$ and $\mathrm{Cu}$ interconnect lines during the early stage of electromigration has been observed using synchrotron polychromatic $\mathrm{x}$ ray microdiffraction. This plastic deformation is characterized by its strong directionality and the measured geometrically necessary dislocation density becomes high enough at test temperature for the dislocation core diffusion to becomes an additional nonnegligible diffusion mechanism. Plasticity-enhanced diffusion may have important consequences in the assessment of device Mean-time-to-failure and reliability with a reinterpretation of the exponent $n$ value in Black's equation.

\section{Acknowledgments:}

The Advanced Light Source is supported by the Director, Office of Science, Office of Basic Energy Sciences, Materials Sciences Division, of the U.S. Department of Energy under Contract No. DE-AC02-05CH11231 at Lawrence Berkeley National Laboratory and University of California, Berkeley, California. The move of the micro-diffraction program from ALS beamline 7.3.3 onto the ALS superbend source 12.3.2 was enabled through the NSF grant \# 0416243. One of the author (NT) thanks Dr. Arief Budiman for useful discussions and sharing some recent results on the dependence of MTF with current density and state of plastic deformation in $\mathrm{Cu}$ interconnects.

\section{References:}

[1] MacDowell, A.A.; Celestre, R.; Chang, C.-H.; Franck, K.; Howells, M.R.; Locklin, S.; Padmore, H.A.; Patel, J.R.; Sandler, R., "Progress toward sub-micron hard X-ray imaging using elliptically bent mirrors", Proceedings of the SPIE - The International Society for Optical Engineering, 3152 (1997) 126-33.

[2] MacDowell, A.A.; Chang, C.H.; Padmore, H.A.; Patel, J.R.; Thompson, A.C., “Grain orientation mapping of passivated aluminum interconnect lines with X-ray microdiffraction”, Mat. Res. Soc. Proc.: Applications of Synchrotron Radiation Techniques to Materials Science IV. Symposium (San Francisco, CA, USA, 13-17 April 1998) 524 (1998) pp. 55-8.

[3] Tamura, N.; Chung, J.-S.; Ice, G.E.; Larson, B.C.; Budai, J.D.; Tischler, J.Z.; Yoon, M.; Williams, E.L.; Lowe, W.P., "Strain and texture in Al-interconnect wires measured by X-ray microbeam diffraction”, Mat. Res. Soc. Proc.: Materials Reliability in Microelectronics IX. Symposium (San Francisco, CA, USA, 6-8 April 1999) 563 (1999) pp 175-80.

[4] Jin-Seok Chung; Tamura, N.; Ice, G.E.; Larson, B.C.; Budai, J.D.; Lowe, W., “X-ray microbeam measurement of local texture and strain in metals”, Mat. Res. Soc. Proc.: Materials Reliability in Microelectronics IX. Symposium (San Francisco, CA, USA, 6-8 April 1999) 563 (1999) 169-74.

[5] Wang PC, Cargill GS, Noyan IC, Hu CK, "Electromigration-induced stress in aluminum conductor lines measured by x-ray microdiffraction”, Applied Physics Letters, 72 (1998) 1296-1298. 
[6] Jin-Seok Chung; Ice, G.E., “Automated indexing for texture and strain measurement with broad-bandpass X-ray microbeams”, Journal of Applied Physics, 86 (1999) pp 5249-55.

[7] Barabash RI, Ice GE, Larson BC, Yang WG, “Application of white X-ray microbeams for the analysis of dislocation structures”, Review of Scientific Instruments, 73 (2002) pp 1652-1654.

[8] Brechbuhl J, Bauch J, Ullrich HJ, "Measurements of residual stresses in micron regions by using synchrotron excited Kossel diffraction”, Crystal Research And Technology, 34 (1999) pp 59-70.

[9] Ice GE, Chung JS, Tischler JZ, Lunt A, Assoufid L , “Elliptical x-ray microprobe mirrors by differential deposition”, Review of Scientific Instruments, 71 (2000) 26352639.

[10] MacDowell, A.A.; Celestre, R.; Chang, C.-H.; Franck, K.; Howells, M.R.; Locklin, S.; Padmore, H.A.; Patel, J.R.; Sandler, R., "Progress toward sub-micron hard X-ray imaging using elliptically bent mirrors", Proceedings of the SPIE - The International Society for Optical Engineering (San Diego, CA, USA, 30-31 July 1997) 3152 (1997) pp 126-33.

[11] Tamura N, Celestre RS, MacDowell AA, Padmore HA, Spolenak R, Valek BC, Chang NM, Manceau A, Patel JR, "Submicron x-ray diffraction and its applications to problems in materials and environmental science”, Review of Scientifc Instruments, 73 (2002) pp. 1369-1372.

[12] N. Tamura, A.A. MacDowell, R. Spolenak, B.C. Valek, J. C. Bravman, W.L. Brown, R. S. Celestre, H.A. Padmore, B.W. Batterman and J.R. Patel, "Scanning X-ray Microdiffraction with submicron white beam for strain/stress and orientation mapping in thin films”, J. of Synchrotron Radiation 10 (2003) 137-14

[13] Martin Kunz, Nobumichi Tamura, Kai Chen, Alastair A. MacDowell, Richard S. Celestre, Matthew M. Church, Sirine Fakra, Edward E. Domning, James M. Glossinger, Dave W. Plate, Brian V. Smith, Tony Warwick, Howard A. Padmore and Ersan Ustundag, "A dedicated superbend x-ray microdiffraction beamline for materials-, geoand environmental sciences at the Advanced Light Source”, Review of Scientific Instruments (2009), in press

[14] Blech I.A., Herring C., “Stress generation by electromigration”, Appl. Phys. Lett., 29 (1976) pp. 131-133

[15] Blech I.A., "Electromigration in Thin Aluminum Films on Titanium Nitride”, Journal of Applied Physics, 47 (1976) pp.1203-1208.

[16] Blech I.A., Tai K.L. , "Measurement of Stress Gradients Generated by Electromigration”, Applied Physics Letters, 30 (1977) 387-389.

[17] Korhonen M. A. , Borgesen P., Tu K. N. , Li C. Y. , “Stress Evolution due to Electromigration in Confined Metal Lines”, Journal of Applied Physics, 73 (1993) pp. 3790-3799.

[18] Valek B.C., Bravman J.C., Tamura N., MacDowell A.A., Celestre R.S., Padmore H.A., Spolenak R., Brown W.L., Batterman B.W., Patel J.R., "Electromigration-induced plastic deformation in passivated metal lines”, Applied Physics Letters, 81 (2002) pp. 4168-4170.

[19] Valek B.C., Tamura N., Spolenak R., Caldwell W.A., MacDowell A.A., Celestre R.S., Padmore H.A., Braman J.C., Batterman B.W., Nix W.D., Patel J.R., "Early stage of 
plastic deformation in thin films undergoing electromigration”, Journal of Applied Physics, 94 (2003) pp. 3757-3761.

[20] Kai Chen; Tamura, N.; Valek, B.C.; Tu, K.N., "Plastic deformation in $\mathrm{Al}(\mathrm{Cu})$ interconnects stressed by electromigration and studied by synchrotron polychromatic Xray microdiffraction”, Journal of Applied Physics, 104 (2008) pp. 013513-1-6.

[21] Hu C. K., Rosenberg R., Lee K.Y., "Electromigration path in Cu thin-film lines", Applied Physics Letters, 74 (1999) pp. 2945-2947.

[22] Spolenak, R.; Barr, D.L.; Gross, M.E.; Evans-Lutterodt, K.; Brown, W.L.; Tamura, N.; Macdowell, A.A.; Celestre, R.S.; Padmore, H.A.; Valek, B.C.; Bravman, J.C.; Flinn, P.; Marieb, T.; Keller, R.R.; Batterman, B.W.; Patel, J.R., "Microtexture and strain in electroplated copper interconnects”, Mat. Res. Soc. Proc.: Materials, Technology and Reliability for Advanced Interconnects and Low-k Dielectrics. Symposium (San Francisco, CA, USA, 23-27 April 2000) 612 (2001) 1-7.

[23] Spolenak, R.; Tamura, N.; Patel, J.R., "X-ray microdiffraction as a probe to reveal flux divergences in interconnects”, AIP Conference Proceedings, 816 (2006) pp. 288-95. [24] Budiman, A. S., Nix, W. D., Tamura, N., Valek, B. C., Gadre, K., Maiz, J., Spolenak, R., Patel, J. R., "Crystal plasticity in Cu damascene interconnect lines undergoing electromigration as revealed by synchrotron x-ray microdiffraction" Applied Physics Letters, 88 (2006) article nr 233515. [25] Budiman, A. S., Besser P.r., Hau-Riege C.S., Marathe A., Joo Y.C., Tamura N., Patel J.R., Nix W.D., "Electromigration-Induced Plasticity: Texture Correlation and Implications for Reliability Assessment”, Journal of Electronic Materials, 38 (2009) pp. 379-391

[26] Budiman, A.S., personal communication. 